\title{
T-duality and non-geometric solutions from double geometry
}

\author{
Martin Cederwall \\ Dept. of Fundamental Physics \\ Chalmers University of Technology \\ SE 41296 Gothenburg, Sweden
}

\begin{abstract}
Although the introduction of generalised and extended geometry has been motivated mainly by the appearance of dualities upon reductions on tori, it has until now been unclear how (all) the duality transformations arise from first principles in extended geometry. A proposal for solving this problem is given in the framework of double field theory. It is based on a clearly defined extension of the definition of gauge symmetry by isometries of an underlying pseudo-Riemannian manifold. The ensuing relation between transformations of coordinates and fields, which is now derived from first principles, differs from earlier proposals.
\end{abstract}

email: martin.cederwall@chalmers.se 


\section{INTRODUCTION}

The development of extended geometry has been motivated mainly by the symmetry enhancement in string theory or M-theory when the models are dimensionally reduced on tori. The T-duality groups $O(d, d ; \mathbb{Z})$ and U-duality groups $E_{n(n)}(\mathbb{Z})$ are expected to arise as geometric (in a generalised sense) symmetries of enlarged tori in doubled geometry [1-22] and exceptional geometry [23-38], respectively.

Much progress has been made in extended geometry, including results concerning global questions [16-22]. The complete procedure to obtain the duality groups from first geometric principles (i.e., as "generalised isometries" of certain backgrounds) has however not been worked out, and it has therefore not even been clear that the symmetry is present. It has been one of the main issues in extended geometry how to derive (all) the duality symmetries. A closely connected and equally urgent question is to what extent extended geometry is able to reproduce non-geometric solutions. In both cases some educated guesses have been needed for the actual transformations and holonomies, which have then been constructed "by hand". The purpose of the present paper is to give a geometric framework for double geometry where these questions are resolved.

In Section 2, relevant aspects of double geometry and double diffeomorphisms are reviewed. Section 3 explains the problem, both in principle and using a few examples. In Section 4, an appropriate enlargement of the group of double diffeomorphisms is proposed, and it is demonstrated how this leads to a resolution of the problems. Section 5 contains a brief discussion and some outlook.

\section{Generalised DifFeOmorphisms in DOUBle FiELD THEORY}

The traditional formulation of generalised diffeomorphisms relies on the introduction of an algebraically invariant tensor of the continuous version of the duality group. In the case of T-duality and double field theory, this tensor is an $O(d, d)$-invariant tensor $\eta_{M N}$. Generators of infinitesimal generalised diffeomorphisms (acting on double covectors) then are

$$
\mathscr{L}_{\xi}=\xi+a-a^{T}=L_{\xi}-a^{T},
$$

where $\xi$ is the translational term given by the vector field $\xi=\xi^{M} \partial_{M}$, and $a_{M}{ }^{N}=\partial_{M} \xi^{N}$. The transpose is defined by the invariant metric as $\left(a^{T}\right)_{M^{N}}=\eta_{M P} \eta^{N Q} a_{Q}{ }^{P}=\left(\eta a^{t} \eta^{-1}\right)_{M^{N}}$ 
Cederwall: "T-duality and non-geometric solutions..." $\ldots \ldots \ldots \ldots \ldots \ldots \ldots \ldots \ldots \ldots \ldots . \ldots$

$\left((\ldots)^{t}\right.$ denoting ordinary transpose), so $a-a^{T}$ is an element of $s o(d, d)$. The commutator of two generalised diffeomorphisms yields

$$
\left[\mathscr{L}_{\xi}, \mathscr{L}_{\chi}\right]=\mathscr{L}_{\llbracket \xi, \chi \rrbracket}
$$

with $\llbracket \xi, \chi \rrbracket=\frac{1}{2}\left(\mathscr{L}_{\xi} \chi-\mathscr{L}_{\chi} \xi\right)$, provided that a section condition holds. This condition is

$$
\eta^{M N} \partial_{M} \otimes \partial_{N}=0
$$

where the notation indicates that the two derivatives may act on any field or gauge parameter. A solution to the section condition is given by the choice of a set of light-like directions on which the fields do not depend.

The duality group one wants to obtain is $O(d, d ; \mathbb{Z})$. In a suitable (split) basis, where coordinates are $\left(x^{m}, \tilde{x}_{m}\right)$ and the invariant metric has the form

$$
\eta_{M N}=\left[\begin{array}{ll}
0 & \mathbb{1} \\
\mathbb{1} & 0
\end{array}\right]
$$

the section condition is solved by demanding $\frac{\partial}{\partial \tilde{x}_{m}}=0$ on fields and parameters. This means that

$$
a-a^{T}=\left[\begin{array}{ll}
\alpha & \beta \\
0 & 0
\end{array}\right]-\left[\begin{array}{ll}
0 & \beta^{t} \\
0 & \alpha^{t}
\end{array}\right]=\left[\begin{array}{cc}
\alpha & \beta-\beta^{t} \\
0 & -\alpha^{t}
\end{array}\right] .
$$

The exponentiation of such a matrix, is an element a

$$
J=\left[\begin{array}{cc}
A & B \\
0 & \left(A^{-1}\right)^{t}
\end{array}\right] \in O(d, d)
$$

where $A B^{t}+B A^{t}=0$. A generic matrix $J \in O(d, d)$ takes the form

$$
J=\left[\begin{array}{ll}
A & B \\
C & D
\end{array}\right]
$$

where $A D^{t}+B C^{t}=\mathbb{1}, A B^{t}+B A^{t}=0$ and $C D^{t}+D C^{t}=0$. Matrices with vanishing lower left corner $(C=0)$ belong to $G L(d) \ltimes \Lambda_{2} \subset O(d, d), \Lambda_{2}$ being the $\frac{d(d-1)}{2}$-dimensional module of 2 -forms. They are precisely those that do not change the solution to the section condition. Solutions to the section condition are parametrised by a pure spinor of $O(d, d)$, belonging (up to a real scale) to the coset $O(d, d) /\left(G L(d) \ltimes \Lambda_{2}\right.$ ). 
$4 \ldots \ldots \ldots \ldots \ldots \ldots \ldots \ldots \ldots$ Cederwall: "T-duality and non-geometric solutions..."

A finite generalised diffeomorphism is of course not given simply by the exponentiation of eq. (2.5), but rather leads to the relation

$$
V_{M}^{\prime}\left(X^{\prime}\right)=e^{-\xi}\left(e^{\mathscr{L}_{\xi}}\right)_{M}{ }^{N} V_{N}(X) \equiv G_{M}^{N}\left(X^{\prime}, X\right) V_{N}(X)
$$

Clearly no lower left entry can be contained in the transformation matrix $G \in O(d, d)$.

In ref. [16], an explicit form of the finite transformations was conjectured, which reads

$$
\begin{aligned}
V_{M}^{\prime}\left(X^{\prime}\right) & =F_{M}^{N} V_{N}(X), \\
F(M) & =\frac{1}{2}\left[M\left(M^{-1}\right)^{T}+\left(M^{-1}\right)^{T} M\right],
\end{aligned}
$$

where

$$
M_{M}^{N}=\frac{\partial X^{N}}{\partial X^{\prime M}} .
$$

It was later shown $[17,18]$ that this expression is correct. The situation deserves some discussion. It was noted in ref. [18] that the commutator of generalised diffeomorphisms may be written in an interesting way as

$$
\left[\mathscr{L}_{\xi}, \mathscr{L}_{\chi}\right]=\mathscr{L}_{[\xi, \chi]}+\Delta_{\xi, \chi}
$$

The bracket $[\cdot, \cdot]$ is the ordinary Lie bracket of vector fields, and the "extra term" $\Delta_{\xi, \chi}$ has the simple form

$$
\Delta_{\xi, \chi}=-a b^{T}+b a^{T}
$$

with $a_{M}{ }^{N}=\partial_{M} \xi^{N}, b_{M}^{N}=\partial_{M} \chi^{N}$. It is then clear that the transformation $\Delta_{\xi, \chi}$ itself represents a generalised diffeomorphism, with parameter $\zeta^{M}=-\xi^{N}\left(b^{T}\right)_{N}{ }^{M}+\eta^{M N} \partial_{N} \lambda$. The transformation $\mathscr{L}_{\zeta}=\Delta_{\xi, \chi}$ has no translational term and does not affect the coordinates. In the basis used above it takes the form

$$
\Delta=\left[\begin{array}{cc}
0 & \beta-\beta^{t} \\
0 & 0
\end{array}\right]
$$

This element of $O(d, d)$ is nilpotent, and exponentiates to $e^{\Delta}=\mathbb{1}+\Delta$. Such pure $b$-field transformations form an ideal of the generalised diffeomorphisms, namely the one preserving $\frac{\partial X}{\partial X^{\prime}}=\mathbb{1}$. This means that given any coordinate transformation matrix $M$, defined by eq. (2.10), there is an equivalence class $[F(M)]$ of transformation matrices acting on tensors under generalised diffeomorphisms, and this class is generated by the representative $F(M)$ of eq. (2.9) by $\Delta$-transformations in the ideal. The matrix $F(M)$ is a canonical representative 
Cederwall: "T-duality and non-geometric solutions..." $\ldots \ldots \ldots \ldots \ldots \ldots \ldots \ldots \ldots \ldots \ldots$.

in the sense that it is the only element in the class expressible as an algebraic function of $M$.

The presence of the $\Delta$ transformations leads to an abelian gerbe structure, described in detail in ref. [18]: although the map $M \rightarrow[F(M)]$ is a group homomorphisms (i.e., $[F(M) F(N)]=[F(M N)])$, the map $M \rightarrow F(M)$ is not, so that

$$
F(M) F(N)=F(M N) e^{\Delta(M, N)}
$$

Since the matrix $M$, due to the section condition, has the form

$$
M=\left[\begin{array}{cc}
m & n \\
0 & \mathbb{1}
\end{array}\right]
$$

it is straightforward to check that insertion in eq. (2.9) for the transformation matrix $F$ also gives a matrix in $O(d, d)$ of the restricted form $(2.6)$, i.e., with vanishing lower left corner.

\section{Statement of the PROBlem}

The situation described above is serious if one wants to understand T-duality in terms of generalised diffeomorphisms, and if one wants to describe non-geometric solutions with holonomy in the T-duality group $O(d, d ; \mathbb{Z})$. The duality group should not be thought of as a global symmetry, a priori built into the formalism, but as a generalised isometry arising for specific solutions, extending the way the mapping class group $S L(d ; \mathbb{Z})$ arises as the discrete isometry group of a torus in ordinary geometry. The problem is that finite generalised diffeomorphisms, as described above, do not fill out the whole of $O(d, d ; \mathbb{Z})$. Similarly, if we consider a doubled torus fibered over some manifold (in the simplest case a circle), the holonomies will always be elements in $O(d, d ; \mathbb{Z})$ of the form $(2.6)$, belonging to the subgroup $\left(G L(d) \ltimes \Lambda_{2}\right)(\mathbb{Z})$.

Concerning the question of dualities, there is no need to illustrate with examples; it is just obvious that some modification is needed if the full T-duality group is to arise as generalised isometries. This modification will be given in the following Section.

For the holonomy, I will illustrate with two examples. They are to different extents non-geometric, and are deliberately chosen identical or similar to examples in ref. [20], so that the resolution in the following Section becomes clear. 
The first example is obtained by two T-dualities, in the $x$ and $y$ directions, of a field configuration with $N$ units of quantised 3 -form flux on a 3 -torus with radii $R_{1,2,3}$. This gives the field configuration

$$
\begin{aligned}
d s^{2} & =f(z)\left(\frac{d x^{2}}{R_{1}^{2}}+\frac{d y^{2}}{R_{2}^{2}}\right)+R_{3}^{2} d z^{2} \\
b_{12} & =-\frac{N z}{2 \pi R_{1}^{2} R_{2}^{2}} f(z)
\end{aligned}
$$

where the function $f(z)$ is given by

$$
f(z)=\frac{1}{1+\left(\frac{N z}{2 \pi R_{1} R_{2}}\right)^{2}}
$$

Such non-geometric solutions were constructed in ref. [39] and discussed from the perspective of generalised geometry in ref. [20]. The (inverse) generalised metric $G^{M N}$ for the $x$ and $y$ directions encoding this metric and $b$-field is

$$
G^{M N}=\left[\begin{array}{cc}
g^{-1} & -g^{-1} b \\
b g^{-1} & g-b g^{1} b
\end{array}\right]=\left[\begin{array}{cccc}
\frac{R_{1}^{2}}{f(z)} & 0 & 0 & \frac{N z}{2 \pi R_{2}^{2}} \\
0 & \frac{R_{2}^{2}}{f(z)} & -\frac{N z}{2 \pi R_{1}^{2}} & 0 \\
0 & -\frac{N z}{2 \pi R_{1}^{2}} & \frac{1}{R_{1}^{2}} & 0 \\
\frac{N z}{2 \pi R_{2}^{2}} & 0 & 0 & \frac{1}{R_{2}^{2}}
\end{array}\right]
$$

The question now is whether this configuration can be constructed in double geometry. Consider going around the $z$-circle from $z=0$ to $z=2 \pi$. We have

$$
\begin{aligned}
G^{-1}(0) & =\left[\begin{array}{cccc}
R_{1}^{2} & 0 & 0 & 0 \\
0 & R_{2}^{2} & 0 & 0 \\
0 & 0 & \frac{1}{R_{1}^{2}} & 0 \\
0 & 0 & 0 & \frac{1}{R_{2}^{2}}
\end{array}\right], \\
G^{-1}(2 \pi) & =\left[\begin{array}{cccc}
R_{1}^{2}+\frac{N^{2}}{R_{2}^{2}} & 0 & 0 & \frac{N}{R_{2}^{2}} \\
0 & R_{2}^{2}+\frac{N^{2}}{R_{1}^{2}} & -\frac{N}{R_{1}^{2}} & 0 \\
0 & -\frac{N}{R_{1}^{2}} & \frac{1}{R_{1}^{2}} & 0 \\
\frac{N}{R_{2}^{2}} & 0 & 0 & \frac{1}{R_{2}^{2}}
\end{array}\right] .
\end{aligned}
$$


It is easily checked that $G^{-1}(2 \pi)=\mathscr{M}^{T} G^{-1}(0) \mathscr{M}$, where $\mathscr{M} \in O(d, d ; \mathbb{Z})$ is the matrix

$$
\mathscr{M}=\left[\begin{array}{cccc}
1 & 0 & 0 & 0 \\
0 & 1 & 0 & 0 \\
0 & -N & 1 & 0 \\
N & 0 & 0 & 1
\end{array}\right]
$$

This matrix is not of the restricted form discussed above, but instead it has vanishing upper right corner. After relabeling the two sets of coordinates $x \leftrightarrow \tilde{x}, \mathscr{M}$ has the form of eq. (2.6) and can be seen as a generalised diffeomorphism. Considering fluctuations around this configuration, however, fields have to depend only on $\tilde{x}$ and not on $x$, since the same solution to the section condition has to apply for fields and gauge transformations.

The second, more genuinely non-geometric, class of configurations has a holonomy implying a change $\tau \rightarrow-\frac{1}{\tau}$ of the complex structure of the 2 -torus. It is not constructed to be a true solution but should be valid as an off-shell configuration. More general such configurations are considered in refs. [40,20], but for our purpose it is enough to let the $b$-field be zero. Consider any metric on the 2 -torus which depends on $z$ with a period $4 \pi$ and has $g(z+2 \pi)=g^{-1}(z)$. The holonomy for the generalised metric is then given by the element

$$
\mathscr{M}=\left[\begin{array}{cc}
0 & \mathbb{1} \\
\mathbb{1} & 0
\end{array}\right] \in O(2,2 ; \mathbb{Z})
$$

Such a T-duality transformation, interchanging $x$ and $\tilde{x}$, can not be obtained as a generalised diffeomorphism, and is therefore not acceptable as holonomy. I will show how this situation may be remedied.

\section{Solution: A geometric proposal for the gauge Symmetries}

The main point of the present paper is to provide a definition of gauge transformations that allows for the reinstatement of the lower left corner of holonomies and of generalised isometries. In order to achieve this it will be necessary to find some way of modifying the concept of gauge transformations, i.e., generalised diffeomorphisms, so that such elements arise.

Recently, it was shown [19] that the invariant tensor $\eta_{M N}$ may be given a geometric rôle. It can be replaced by any pseudo-Riemannian metric $H_{M N}$ (with split signature), defining a non-dynamical pseudo-Riemannian background geometry on the doubled manifold. The only restriction (which in itself is quite strong) is the possibility to introduce a section condition. 
This effectively imposes a flatness condition in at least half the directions, corresponding to the $\tilde{x}$ 's.

Derivatives are replaced by covariant derivatives containing the torsion-free affine connection compatible with $H$. The generators then take the form

$$
\mathscr{L}_{\xi}=\xi^{M} D_{M}+a-a^{T}=L_{\xi}-a^{T}
$$

where now $a_{M}{ }^{N}=D_{M} \xi^{N}$ and $\left(a^{T}\right)_{M^{N}}=H_{M P} H^{N Q} a_{Q}{ }^{P}$. These transformations are generically inequivalent to eq. (2.1), but are of course equivalent if $H$ is flat. The closure of the algebra (more precisely, algebroid) relies on the first Bianchi identity (the torsion Bianchi identity) together with a covariant section condition $H^{M N} D_{M} \otimes D_{N}=0$, and there are no curvature obstructions. It puts no further restrictions on curvature than the partial flatness condition already mentioned. The metric $H$ is a non-dynamical defining background metric parametrising the embedding $O(d, d) \subset G L(2 d)$, and has nothing to do with the (dynamical) generalised metric in the coset $O(d, d) /(O(d) \times O(d))$.

Even if the definitions (2.1) and (4.1) of the generators coincide for a flat defining metric $H$, the geometric, rather than algebraic, interpretation allows for the possibility to let it transform. Since it is an ordinary metric it will transform as a tensor of ordinary, not generalised diffeomorphisms (being covariantly constant, it is invariant under generalised diffeomorphisms). This may at first sound strange: we already have a way of changing coordinates through generalised diffeomorphisms, and it seems contradictory to have two different types of transformations (and objects behaving like tensors under both) at once. This is certainly true for most ordinary diffeomorphisms, but there is one class which is interesting, namely isometries, leaving the defining metric, and thus the form of generalised diffeomorphisms (4.1), unchanged.

Consider first continuous isometries, generated by $L_{u}$, where $u$ is a Killing vector. The observation that the "covariant" generalised diffeomorphisms are formed from $\xi$ in a manner which is manifestly covariant under ordinary diffeomorphisms leads immediately to

$$
\left[L_{u}, \mathscr{L}_{\xi}\right]=\mathscr{L}_{[u, \xi]}
$$

It is important to note that the Killing vector, unlike the parameter $\xi$, is not constrained to obey the section condition, but has arbitrary coordinate dependence. Such transformations, with Killing vectors possibly depending on both $x$ and $\tilde{x}$ may generate transformations changing the choice of section condition and having non-vanishing lower left corner. 
It is however not continuous isometries that are of most interest, at least not when the goal is to understand discrete duality transformations and holonomy. The manifest covariance of eq. (4.1) means that the behaviour of (infinitesimal or finite) generalised diffeomorphisms under any (finite) isometry is known: they behave like tensors, and transform with the transformation matrix $\mathscr{M}_{M}{ }^{N}=\frac{\partial X^{N}}{\partial X^{\prime M}}$ of the isometry. If the transformation matrix $\mathscr{M}$ is orthogonal (with respect to the metric $H$ ), i.e., if $\mathscr{M}^{T} \mathscr{M}=\mathbb{1}$, it represents an isometry. We would again like to stress that, unlike finite generalised diffeomorphisms, there is no need for parameters to satisfy the section condition. It can be noted that also anti-isometries, with $H \rightarrow-H$ (which is meaningful on a manifold with split signature), are allowed, since $H$ appears only quadratically in $\mathscr{L}_{\xi}$.

The group of isometries $\mathscr{I}$ acts as automorphisms of the original algebra of generalised diffeomorphisms. Some of them may be inner automorphisms, or at least uninteresting, if they correspond to coordinate transformations already realisable as finite generalised diffeomorphisms. Some, on the other hand, may act as outer automorphisms. This applies to any isometry that rotates the solution of the section condition. In ordinary geometry, all isometries act as inner automorphisms, but in doubled geometry, the section condition prevents the generalised diffeomorphisms to rotate its solution. The elements in $\mathscr{I}$ that rotate the solution to the section condition (having entries in the lower left corner) give a geometric tool to realise such transformations, especially in the case of a flat torus, where $\mathscr{I}$ contains the group

$$
S L(2 d ; \mathbb{Z}) \cap O(d, d)=O(d, d ; \mathbb{Z})
$$

which is the isometry subgroup of the mapping class group.

The proposal for a better definition of the gauge transformations should now be obvious. It reads:

- The gauge transformations are declared to be generalised diffeomorphisms, together with their automorphisms.

By elevating the status of isometries, i.e., automorphisms of generalised diffeomorphisms, to gauge symmetries, there are no longer any outer automorphisms. They are used in order to obtain the full duality group, and the full holonomy. On a flat torus, this definition enlarges the gauge symmetries by homotopically non-trivial sectors which were inaccessible in the earlier formulation (but nevertheless sometimes inserted "by hand").

Take an element $\mathscr{M} \in \mathscr{I}$. As shown above, covectors transform as $V \rightarrow \mathscr{M} V$. A transformation matrix changes according to $M \rightarrow \mathscr{M} M \mathscr{M}^{-1}=\mathscr{M} M \mathscr{M}^{T}$, which is consistent with

$$
\begin{aligned}
F(M) & =\frac{1}{2}\left[M\left(M^{-1}\right)^{T}+\left(M^{-1}\right)^{T} M\right] \\
& \rightarrow \frac{1}{2}\left[\mathscr{M} M \mathscr{M}^{T}\left(\left(\mathscr{M} M \mathscr{M}^{T}\right)^{-1}\right)^{T}+\left(\left(\mathscr{M} M \mathscr{M}^{T}\right)^{-1}\right)^{T} \mathscr{M} M \mathscr{M}^{T}\right] \\
& =\mathscr{M} F(M) \mathscr{M}^{T}
\end{aligned}
$$


(this is of course just another way of stating the automorphism property). A consequence of the extended definition of gauge transformations is that the transformation matrix associated with an isometry is $\mathscr{M}$ itself, and not the formal extension $F(\mathscr{M})$ of the relation (2.9) to arbitrary elements in $G L(2 d)$ or $O(d, d)$. In earlier work [20], the latter has been used, leading to $F(\mathscr{M})=\mathscr{M}^{2}$, and in order to guess a coordinate transformation corresponding to a certain holonomy or T-duality transformation, a square root of the transformation matrix was needed. This lead to some peculiar behaviour, which in some cases, like our second example above, included orientation-reversing transformations. This is not the case in the present proposal.

Let us now go back to the problem and to the examples.

Concerning duality transformations in $O(d, d ; \mathbb{Z})$, they are now all present as gauge transformations in a situation with a flat defining metric.

In the first example of a non-geometric field configuration, given by eq. (3.1), it was seen that the problem in a sense could be circumvented by a reinterpretation of the physical subspace (the choice of solutions to the section condition). With the new proposal, this is no longer necessary. The holonomy (3.5) is a discrete gauge transformation disregarding the choice of section condition. The situation is improved, in the sense that fluctuations around this configuration are no longer restricted to depend only on $\tilde{x}$ 's, but can obey any solution to the section condition, including dependence on $x$ 's only (in this patch). Of course, when moving to other patches, the section condition transforms accordingly.

In the second example with holonomy given by eq. (3.6), and any other with generic enough holonomy, the only possibility with the previous understanding was to put in the desired holonomy by hand. In the present picture, there is a geometric prescription for the transformation which makes it a gauge transformation. In the example, the actual coordinate transformation is the exchange of $x$ and $\tilde{x}$, not its square root. Again, the fields in a certain patch must obey some section condition, which then is rotated by the holonomy.

\section{Conclusions}

It has been demonstrated how the gauge symmetries of double field theory may be extended to include isometries of the non-dynamical defining metric. They act as automorphisms of the generalised diffeomorphisms, and the important part consists of the outer automorphisms. An analogous statement should be true for exceptional geometry, i.e., extended geometry in the context of U-duality, based on exceptional groups. In those cases, the geometric interpretation of the invariant tensors, corresponding to the results of ref. [19], has not yet been worked out (the interpretation of the invariant tensor as a metric tensor is particular to doubled geometry). 
Since the section condition, with the earlier definition of gauge symmetries, has been responsible for the restriction of the transformation matrices, a belief has sometimes been expressed that recovery of the full duality group might require a relaxation of the section condition. This turned out not to be the case. However, it is clear that the present construction requires the solution to the section condition to be multiple valued (for non-geometric field configurations), and subject to transformations including the holonomy described earlier.

An possible objection to the present construction is that it provides more of a patching up of the problematic situation than a radical solution. Although the construction has a firm geometric basis, this may be so, and if there is a better one, it is likely to arise in a context where the section condition is abandoned altogether, and instead arises dynamically. Suppose for example that a solution to the section condition is imposed by the choice of a pure spinor. These variables must then locally be pure gauge degrees of freedom. If such a formulation exists, it should probably contain (ordinary) general coordinate invariance as an essential ingredient.

Acknowledgements: The author wishes to thank David Berman, Jeong-Hyuck Park, Daniel Waldram, Lisa Carbone, and especially Olaf Hohm, for discussions.

\section{REFERENCES}

[1] M.J. Duff, "Duality rotations in string theory", Nucl. Phys. B335 (1990) 610.

[2] A.A. Tseytlin, "Duality symmetric closed string theory and interacting chiral scalars", Nucl. Phys. B350 (1991) 395.

[3] W. Siegel, "Two vierbein formalism for string inspired axionic gravity", Phys. Rev. D47 (1993) 5453 arXiv:hep-th/9302036.

[4] W. Siegel, "Superspace duality in low-energy superstrings", Phys. Rev. D48 (1993) 2826 [arXiv:hep-th/ 9305073].

[5] W. Siegel, "Manifest duality in low-energy superstrings", in Berkeley 1993, Proceedings, Strings '93 353 arXiv:hep-th/9308133.

[6] C.M. Hull, "A geometry for non-geometric string backgrounds", J. High Energy Phys. 0510 (2005) 065 arXiv:hep-th/0406102.

[7] C.M. Hull, "Doubled qeometry and T-folds", J. High Energy Phys. 0707 (2007) 080 [arXiv:hep-th/ 0605149].

[8] C. Hull and B. Zwiebach, "Double field theory", J. High Energy Phys. 0909 (2009) 99 arXiv:0904.4664 [hep-th]].

[9] O. Hohm, C.M. Hull and B. Zwiebach, "Background independent action for double field theory", J. High Energy Phys. 1007 (2010) 016 arXiv:1003.5027 [hep-th]].

[10] O. Hohm, C.M. Hull and B. Zwiebach, "Generalized metric formulation of double field theory", J. High Energy Phys. 1008 (2010) 008 [arXiv:1006.4823 [hep-th]]. 
[11] O. Hohm and S.K. Kwak, "Frame-like geometry of double field theory", J. Phys. A44 (2011) 085404 [arXiv:1011.4101 [hep-th]].

[12] O. Hohm and S.K. Kwak. "N=1 supersymmetric double field theory", J. High Energy Phys. 1203 (2012) 080 arXiv:1111.7293 [hep-th]].

[13] I. Jeon, K. Lee and J.-H. Park, "Differential geometry with a projection: Application to double field theory", J. High Energy Phys. 1104 (2011) 014 arXiv:1011.1324 [hep-th]].

[14] I. Jeon, K. Lee and J.-H. Park, "Stringy differential geometry, beyond Riemann", Phys. Rev. D84 (2011) 044022 arXiv:1105.6294 [hep-th]].

[15] I. Jeon, K. Lee and J.-H. Park, "Supersymmetric double field theory: stringy reformulation of supergravity", Phys. Rev. D85 (2012) 081501 arXiv:1112.0069 [hep-th]].

[16] O. Hohm and B. Zwiebach, "Large gauge transformations in double field theory", J. High Energy Phys. 1302 (2013) 075 arXiv:1207.4198 [hep-th]].

[17] J.-H. Park. "Comments on double field theory and diffeomorphisms". J. High Energy Phys. 1306 (2013) 098 arXiv:1304.5946 [hep-th]].

[18] D.S. Berman, M. Cederwall and M.J. Perry, "Global aspects of double geometry", J. High Energy Phys. 1409 (2014) 66 [arXiv:1401.1311 [hep-th]].

[19] M. Cederwall, "The qeometry behind double qeometry", J. High Energy Phys. 1409 (2014) 70 [arXiv: 1402.2513 [hep-th]].

[20] O. Hohm, D. Lüst and B. Zwiebach, "The spacetime of double field theory: Review, remarks and outlook", Fortsch. Phys. 61 (2013) 926 [arXiv:1309.2977 [hep-th]].

[21] G. Papadopoulos, "Seeking the balance: Patching double and exceptional field theories", arXiv:1402.2586 [hep-th].

[22] C.M. Hull, "Finite gauge transformations and geometry in double field theory", arXiv:1406.7794 [hep-th].

[23] C.M. Hull, "Generalised qeometry for M-theory", J. High Energy Phys. 0707 (2007) 079 [arXiv:hep-th/ 0701203].

[24] P.P. Pacheco and D. Waldram, "M-theory, exceptional generalised geometry and superpotentials", J. High Energy Phys. 0809 (2008) 123 arXiv:0804.1362 [hep-th]].

[25] C. Hillmann, "Generalized $E_{7(7)}$ coset dynamics and $D=11$ supergravity", J. High Energy Phys. 0903 (2009) 135 [arXiv:0901.1581 [hep-th]].

[26] D.S. Berman and M.J. Perry, "Generalised geometry and M-theory", J. High Energy Phys. 1106 (2011) 074 arXiv:1008.1763 [hep-th]].

[27] D.S. Berman, H. Godazgar and M.J. Perry, "SO(5,5) duality in M-theory and generalized geometry", Phys. Lett. B700 (2011) 65 [arXiv:1103.5733 [hep-th]].

[28] D.S. Berman, H. Godazgar, M. Godazgar and M.J. Perry, "The local symmetries of M-theory and their formulation in generalised geometry", J. High Energy Phys. 1201 (2012) 012 arXiv:1110.3930 [hep-th]].

[29] D.S. Berman, H. Godazgar, M.J. Perry and P. West, "Duality invariant actions and generalised geometry", J. High Energy Phys. 1202 (2012) 108 arXiv:1111.0459 [hep-th]].

[30] A. Coimbra, C. Strickland-Constable and D. Waldram, " $E_{d(d)} \times \mathbb{R}^{+}$qeneralised geometry, connections and $M$ theory", J. High Energy Phys. 1402 (2014) 054 arXiv:1112.3989 [hep-th]].

[31] A. Coimbra, C. Strickland-Constable and D. Waldram, "Supergravity as generalised geometry II: $E_{d(d)} \times$ $\mathbb{R}^{+}$and $M$ theory", J. High Energy Phys. 1403 (2014) 019 arXiv:1212.1586 [hep-th]].

[32] D.S. Berman, M. Cederwall, A. Kleinschmidt and D.C. Thompson, "The gauge structure of generalised diffeomorphisms", J. High Energy Phys. 1301 (2013) 64 [arXiv:1208.5884 [hep-th]].

[33] J.-H. Park and Y. Suh, "U-geometry: SL(5)", J. High Energy Phys. 1406 (2014) 102 arXiv:1302.1652 [hep-th]].

[34] M. Cederwall, J. Edlund and A. Karlsson, "Exceptional qeometry and tensor fields", J. High Energy Phys. 1307 (2013) 028 arXiv:1302.6736 [hep-th]]. 
Cederwall: "T-duality And non-Geometric solutions..." $\ldots \ldots \ldots \ldots \ldots \ldots \ldots \ldots \ldots \ldots \ldots \ldots 13$

[35] M. Cederwall, "Non-gravitational exceptional supermultiplets", J. High Energy Phys. 1307 (2013) 025 arXiv:1302.6737 [hep-th]].

[36] M. Cederwall, "M-branes on U-folds", in proceedings of 7th International Workshop "Supersymmetries and Quantum Symmetries" Dubna, 2007 [arXiv:0712.4287 [hep-th]].

[37] O. Hohm and H. Samtleben, "Exceptional field theory I: $E_{6(6)}$ covariant form of M-theory and type IIB", Phys. Rev. D89 (2014) 066016 arXiv:1312.0614 [hep-th]].

[38] O. Hohm and H. Samtleben, "Exceptional field theory II: $E_{7(7)}$ ", Phys. Rev. D89 (2014) 066016 [arXiv: 1312.4542 [hep-th]].

[39] S. Kachru, M.B. Schulz, P.K. Tripathy and S.P. Trivedi, "New supersymmetric string compactifications", J. High Energy Phys. 0303 (2003) 061 arXiv:hep-th/0211182.

[40] C. Condeescu, I. Florakis, C. Kounnas and D. Lüst, "Gauged supergravities and non-geometric $Q / R$-fluxes from asymmetric orbifold CFT's", J. High Energy Phys. 1310 (2013) 057 [arXiv:1307.0999 [hep-th]]. 